\title{
Chemical Composition and Physicochemical Analysis of Opuntia dillenii Extracts Grown in Morocco
}

\author{
EL Hassania Loukili (D), ${ }^{1}$ Farid Abrigach, ${ }^{1}$ Mohamed Bouhrim (iD), ${ }^{2}$ Mohamed Bnouham (iD), \\ Marie-laure Fauconnier, ${ }^{3}$ and Mohammed Ramdani ${ }^{1}$ \\ ${ }^{1}$ Laboratory of Applied and Environmental Chemistry (LCAE), Mohammed First University, \\ Oujda, Morocco \\ ${ }^{2}$ Laboratory of Bioresources Biotechnology Ethnopharmacology and Health, Department of Biology, Faculty of Sciences, \\ University Mohamed Ist, Oujda, Morocco \\ ${ }^{3}$ Laboratory of Chemistry of Natural Molecules, University of Liège, Gembloux Agro-Bio Tech, 2 Passage of Déportés B-5030, \\ Gembloux, Belgium
}

Correspondence should be addressed to EL Hassania Loukili; e.loukili@ump.ac.ma

Received 21 September 2020; Revised 16 December 2020; Accepted 22 December 2020; Published 5 January 2021

Academic Editor: Toyonobu Usuki

Copyright (c) 2021 EL Hassania Loukili et al. This is an open access article distributed under the Creative Commons Attribution License, which permits unrestricted use, distribution, and reproduction in any medium, provided the original work is properly cited.

The chemical composition and physicochemical properties of hexane and ethyl acetate extracts of skin, juice, and seeds of Opuntia dillenii fruit collected from three Moroccan regions (Oujda, Nador, and Essaouira) were studied. The study revealed that the seed oil extracts presented the highest yield of $13.12 \%$, followed by the skin fraction (1.77\%) and the juice extract $(0.49 \%)$. The evaluation of fatty acid compositions using GC-MS analysis revealed the presence of linoleic acid as a dominating unsaturated fatty acid with a value of $72.39 \%$, followed by palmitic acid, oleic acid, and stearic acid in all localities. Otherwise, the juice extract of Oujda locality was richer in margaric acid (37.41\%), followed by Essaouira skin extract (10.7\%) and Oujda seed extract (6.18\%). However, the campesterol was detected only in trace in the juice extract. The physicochemical properties of $O$. dillenii seed oils such as acid value, peroxide value, ester value, $\mathrm{pH}$ value, saponification value, density, and refractive index were all found to be in good agreement with the quality criteria for pure and fresh oils. In addition, principal component analysis (PCA) and hierarchical cluster analysis (HCA) were implemented to compare the difference in the chemical composition of the different $O$. dillenii extracts.

\section{Introduction}

Opuntia dillenii, commonly known as prickly pear, is a perennial plant belonging to the Cactaceae, Caryophyllales, family and the Caryophyllidae subclass $[1,2]$. It is extensively distributed in the desert, semidesert, and dry regions in the tropics and subtropics [3]. This plant is native to tropical America and India. However, it can be grown in Australia, Asia, and the Mediterranean region [4]. It was introduced in Europe and North Africa in the $16^{\text {th }}$ century [5]. Cactus has remarkable benefits to human and animal health [6]. It can be a source of various chemical compounds of biological and pharmacological importance.

Several studies have revealed its high efficacy in the treatment of antihyperlipidemic, antiatherosclerotic effects
[7]. Its ability to protect nerve cells from Alzheimer's, Parkinson's, and cerebral vascular diseases has also been reported [8]. Particularly, juice of Opuntia fruits is a rich source of dietary fiber, vitamins (B1, B2, and C), and natural colorants (betanin and indicaxanthin) [9-14], which are responsible for the intense red coloring of the fruit [11]. It is also known by its analgesic, antihyperglycemic [15], inflammatory [16], antifungal [17], anticancer [1, 18], hepatoprotective [19], and antidiabetic activities [18, 20]. Indeed, the seeds of $O$. dillenii may contribute to the higher antioxidant activity due to the high concentrations of polyphenols and flavonoids and the presence of a large amount of unsaturated fatty acids that provide valuable natural antioxidants for the pharmaceutical and food industry [21]. The O. dillenii oil is well-known for its richness 
in essential fatty acids and vitamin E [6] which have efficiency potential on the skin and can be used as antiaging and antiwrinkle agents. The oil of $O$. dillenii could also be a source of linoleic acid (omega-6) [22], which is involved in the regulation of cardiovascular disorders. This oil has also shown a hepatoprotective and antidiabetic effect $[23,24]$. All these properties have been able to give a positive impact on the exploration of $O$. dillenii fruits.

In this paper, continuing our previous work on O. dillenii plant, we report the evaluation of the chemical and physicochemical properties of hexane and ethyl acetate extracts of skin, juice, and fruit seeds of $O$. dillenii grown in three different regions of Morocco (Oujda, Nador, and Essaouira). This investigation was performed to assess the potential nutritional value of $O$. dillenii fruits for human and animal consumption. The extracts were analyzed, characterized, and identified by GC-MS. The variation of $O$. dillenii extracts parameters between the three regions was investigated using statistical analyses (hierarchical cluster analysis (HCA) and principal component analysis (PCA)).

\section{Materials and Methods}

2.1. Chemicals and Reagents. Hexane, ethyl acetate, and methanol were purchased from Merck (Karlsruhe, Germany). All chemicals and solvents were of highly analytical grade and were used as received from Merck without further purification.

2.2. Plant Material. The matured purple fruits of prickly pear (O. dillenii) were collected in February 2017 from three regions in Morocco (Figure 1): Oujda (Sidi Maafa), Nador (Kariat Arkmane), and Essaouira (Smimou). The geographical and climatic data of the studied regions are presented in Table 1. The plant species was authenticated by Prof. M. Bnouham (Faculty of Sciences, University Mohammed Premier, Oujda, Morocco).

The sampled fruits were transported to the laboratory, washed thoroughly with water to remove dust and spines, then peeled, and mixed for 5 min using a Moulinex blender (Faciclic Glass LM310E, Groupe Seb, Mayenne, France) to separate seeds from the juice by passing through a sieve with a $2 \mathrm{~mm}$ screen (Figure 2). The juice was dehydrated by heating at $40^{\circ} \mathrm{C}$ in the oven for 15 days. Seeds and skins were washed with distilled water, dried at room temperature $\left(25^{\circ} \mathrm{C}\right)$, for one week, weighed to calculate the percentage in the edible fraction, then reduced to a fine powder using a Moulinex coffee grinder (DPA241, Groupe Seb, Lourdes, France), and stored at $-20^{\circ} \mathrm{C}$, as well as the juice, for a maximum of eight weeks.

2.3. Preparation of Plant Extracts. Ground seeds, skin, and juice of $O$. dillenii were macerated with hexane as a nonpolar solvent for removal of fatty acids. Each powder $(50 \mathrm{~g})$ was mixed with $110 \mathrm{~mL}$ of hexane. The mixture was stirred at room temperature $\left(18^{\circ} \mathrm{C}\right)$ for $24 \mathrm{~h}$ and filtered through a glass filter crucible $(50 \mathrm{~mL}$, Porosity 4, Isolab, Wertheim, Germany) connected to a water aspirator. The extracts were concentrated on a rotary evaporator (Laborota 4000, Heidolph Instruments, Schwabach, Germany) under reduced pressure at $40^{\circ} \mathrm{C}$ to obtain the corresponding powder which was then used by repeating the process of maceration with ethyl acetate, filtration, and concentration under reduced pressure. The extracts were conserved in dark bottles and stored at $4^{\circ} \mathrm{C}$ until use.

2.4. Physicochemical Analysis of O. dillenii Oil. In order to check the quality and appropriate conservation of the oil samples, the acid value and the ester value (expressed as milligram $\mathrm{KOH}$ per $\mathrm{g}$ of oil) and the peroxide value (PV) (expressed as milliequivalents of active oxygen per $\mathrm{kg}$ of oil) were determined. The water content in the hexane extracts has been calculated according to the standard NF V 05-108 [25]. The acid value, ester value, peroxide value, and saponification value were determined according to the Codex Stan 210-1999 [26] standard protocol. The standard protocols NF ISO 6883 (July 1995) [27] and NF ISO 6320 (January 1996) [25] were adopted to measure the density and refractive index at $20^{\circ} \mathrm{C}$. The density of the oils was determined by a mass over volume measurement. The $\mathrm{pH}$ values were measured using a CPC-501 pH-meter (Elmetron, Chorzow, Poland). The measures were performed in triplicate for each parameter, and the results were presented as mean \pm standard deviation.

2.5. Fatty Acid GC-MS Analysis of O. dillenii Extracts. The fatty acid methyl esters of hexane and ethyl acetate extracts of $O$. dillenii fruits were prepared following the standard protocol NF T60-233 [28]. Their separation and identification were performed on a Shimadzu GC system (Kyoto, Japan) equipped with a BPX25 capillary column with 5\% diphenyl, 95\% dimethylpolysiloxane phase ( $30 \mathrm{~m} \times 0.25 \mathrm{~mm}$ inner diameter $\times 0.25 \mu \mathrm{m}$ film thickness), coupled to a QP2010 MS. Pure helium gas (99.99\%) was used as carrier gas with a constant flow rate of $3 \mathrm{~mL} / \mathrm{min}$. The injection, ion source, and interface temperatures were all set at $250^{\circ} \mathrm{C}$. The temperature program used for the column oven was $50^{\circ} \mathrm{C}$ (held for $1 \mathrm{~min}$ ), heated to $250^{\circ} \mathrm{C}$ at $10^{\circ} \mathrm{C} / \mathrm{min}$ and held for $1 \mathrm{~min}$. The ionization of the sample components was done in the EI mode $(70 \mathrm{eV})$. The mass range scanned was $40-300 \mathrm{~m} / \mathrm{z} .1 \mu \mathrm{L}$ of each prepared extract diluted with an appropriate solvent was injected in a splitless mode (split ratio $90: 1$ ). All samples were analyzed in triplicate. Finally, compounds were identified by comparison of their retention times with those of authentic standards and their mass spectrum fragmentation patterns with those found in databases or those stored on the National Institute of Standards and Technology (NIST) 147, 198 compounds. LabSolutions (version 2.5) was used for data collection and processing.

2.6. Statistical Analysis. All the results are expressed as mean \pm standard deviation (SD). The obtained data of the oil components with a percentage greater than $5 \%$ were analyzed statistically using the principal component (PCA) and 


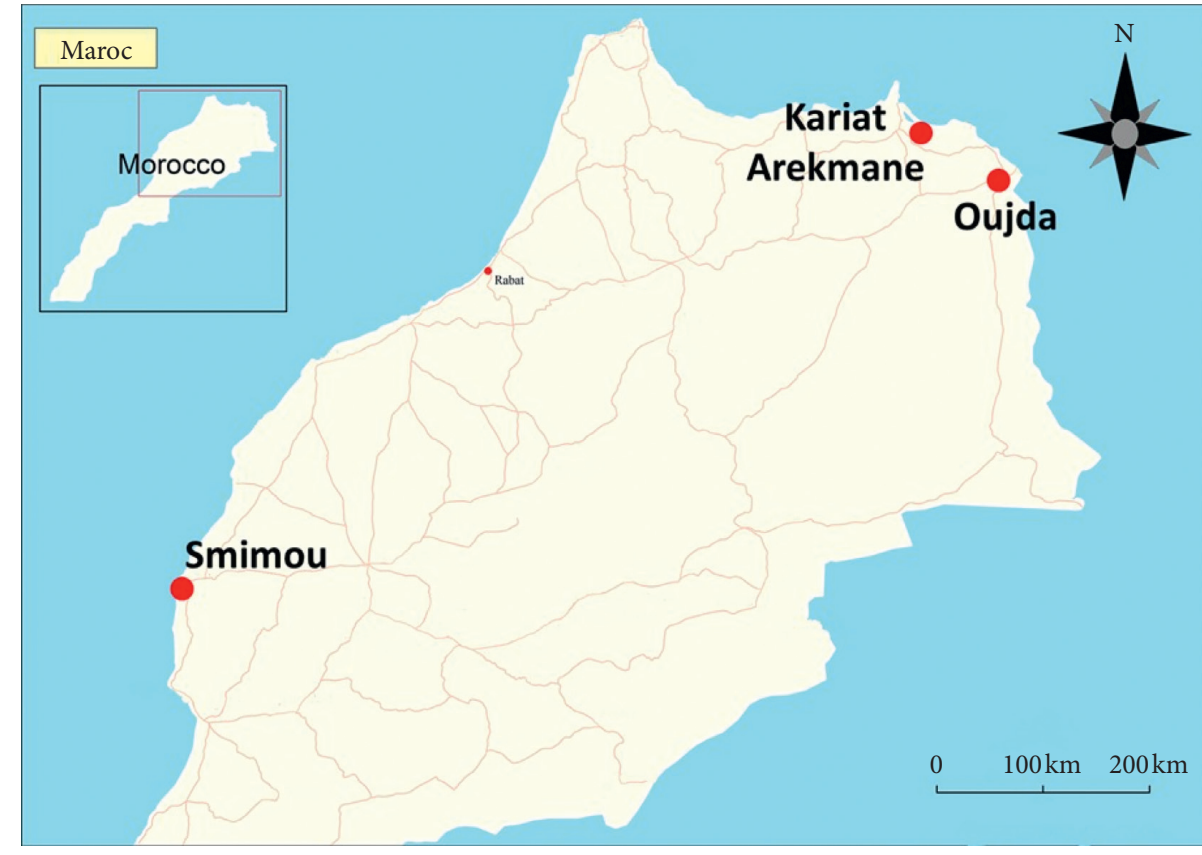

FIGURE 1: Sampling sites: Oujda, Nador, and Essaouira localization.

TABLE 1: Ecogeographic characteristics of the sampling sites.

\begin{tabular}{|c|c|c|c|c|c|c|c|}
\hline Sampling location & Region & Latitude & Longitude & Altitude $(\mathrm{m})$ & MAP $(\mathrm{mm})$ & MWT $\left({ }^{\circ} \mathrm{C}\right)$ & $\operatorname{MST}\left({ }^{\circ} \mathrm{C}\right)$ \\
\hline Sidi Maafa & Oujda & $34^{\circ} 38^{\prime} 16^{\prime}$ & $1^{\circ} 53^{\prime} 16^{\prime}$ & 450 & 338 & 10.7 & 23.9 \\
\hline Kariat Arkmane & Nador & $35^{\circ} 06^{\prime} 15$ & $2^{\circ} 44^{\prime} 04^{\prime}$ & 2 & 313 & 14.5 & 22.8 \\
\hline Smimou & Essaouira & $31^{\circ} 12^{\prime} 49^{\prime}$ & $9^{\circ} 42^{\prime} 21^{\prime}$ & 237 & 300 & 8.7 & 24.5 \\
\hline
\end{tabular}

MAP: mean annual precipitation; MWT: mean of winter temperature; MST: mean of summer temperature. Source: https://fr.weatherspark.com/y/31980/ Météo-habituelle-à-Smimou-Maroc.

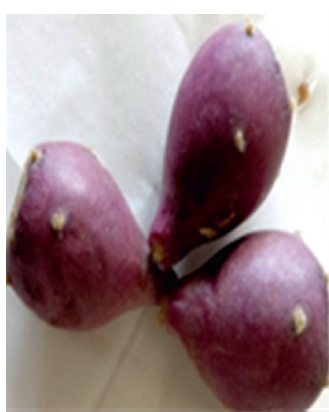

(a)

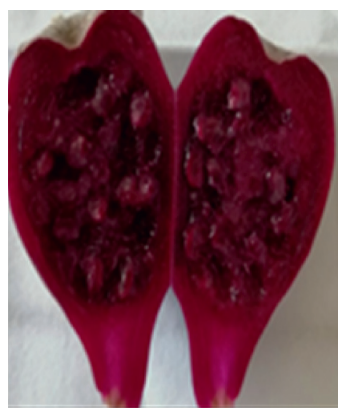

(b)

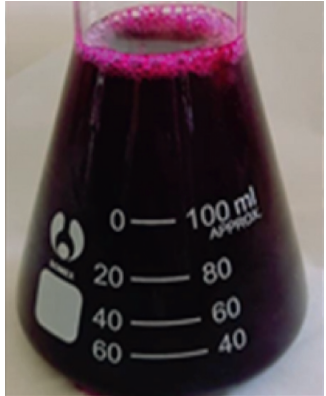

(c)

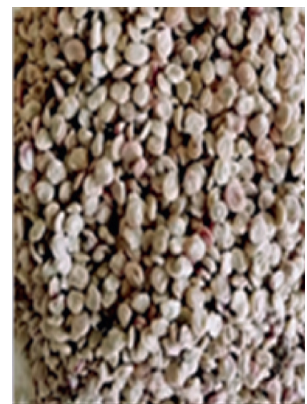

(d)

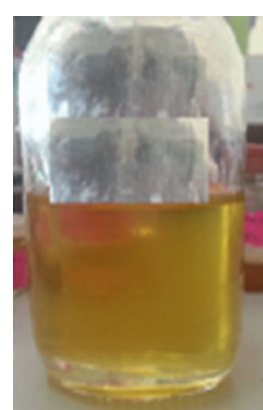

(e)

Figure 2: O. dillenii fruit: (a) fresh fruits; (b) pulp; (c) juice; (d) seeds; (e) seed oil.

hierarchical cluster (HCA) analyses. All the statistics were performed employing the XLSTAT software.

\section{Results and Discussion}

3.1. Yield Percentage of Skin, Pulp, and Seeds in Fruit. The length and width (diameter) as well as the skin, juice, and seed content of the $O$. dillenii fruits, according to their locality, are summarized in Table 2. The morphological study showed that the Essaouira fruits were bigger than those of
Oujda and Nador, while no large differences were observed between the morphological characteristics (length and width) of the $O$. dillenii belonging to Oujda and Nador locality. On the other hand, the percentage yield analysis of each dry part (skin, juice, and seeds) obtained from fresh Essaouira fruits revealed that the seed content was found to be the highest (14.7\%), followed by skin (11.6\%) and juice (4.4\%). The Essaouira fruit contained the highest content of seeds, skin, and juice. In the literature, Ali Alsaad reported lower fruit seed content, 9.5\%, in Iraq [22]. 
TABLE 2: Morphological characteristics, $\mathrm{pH}$ juice, and yield of the dry skin, juice, and seeds.

\begin{tabular}{|c|c|c|c|c|c|c|}
\hline \multirow{2}{*}{ Locality } & \multicolumn{6}{|c|}{ Yield } \\
\hline & Length $(\mathrm{cm})$ & Width $(\mathrm{cm})$ & Dry skin (\%) & Dry juice (\%) & Dry seeds (\%) & $\mathrm{pH}$ (juice) \\
\hline Oujda & $5.00 \pm 0.14$ & $2.50 \pm 0.34$ & $8.50 \pm 0.17$ & $4.00 \pm 0.33$ & $13.30 \pm 0.18$ & $3.90 \pm 0.28$ \\
\hline Nador & $4.70 \pm 0.15$ & $2.30 \pm 0.09$ & $9.16 \pm 0.15$ & $3.03 \pm 0.30$ & $12.80 \pm 0.24$ & $3.20 \pm 0.26$ \\
\hline Essaouira & $7.00 \pm 0.10$ & $4.00 \pm 0.47$ & $11.60 \pm 0.14$ & $4.40 \pm 0.10$ & $14.70 \pm 0.16$ & $4.80 \pm 0.21$ \\
\hline
\end{tabular}

These findings demonstrated clearly that the fresh O. dillenii fruits contain more than $75 \%$ of water, which was in good agreement with previous data reported in the literature [29, 30]. Furthermore, the juice fruit of Essaouira was less acidic compared to that of the two other localities, $\mathrm{pH}=4.8,3.9$, and 3.2 for Essaouira, Oujda, and Nador, respectively.

\subsection{Physical and Chemical Parameters of O. dillenii Seed Oils.} The study of the physicochemical properties of any product is a very useful way to measure its quality before its use or consumption. These different parameters can be influenced by climatic conditions as well as cultural practices. In this context, some physicochemical parameters of $O$. dillenii seed oils (for the three sites) such as acid value, ester value, peroxide value, saponification value, $\mathrm{pH}$ value, density, and refractive index were measured following the appropriate protocols. The obtained results were collected and are presented in Table 3.

According to the obtained data, the water content in seed oil for the three localities of $O$. dillenii was found to be below the max value proposed by Codex Stan 210-1999 and COI standards ( $\max 0.2 \%$ ). The higher content was shown by Nador sample with $0.189 \%$, followed by Essaouira and Oujda samples with values of $0.154 \%$ and $0.148 \%$, respectively. The peroxide value, which is the most used indicator to describe the relative stability to lipid oxidation of oils, was calculated [31]. Values greater than $10 \mathrm{meq} / \mathrm{kg}$ are highly susceptible to autooxidation as a consequence of moisture or presence of trace elements [32]. In our case, the peroxide values of the three localities ranged from 5.60 to 6.35 , less than 10 meq $\mathrm{O}_{2} / \mathrm{kg}$ of oil. These outcomes suggested that our oils possess a high shelf life. However, these values are higher than those obtained by El Mannoubi et al. [33] for O. ficus-indica seed oil and by Alsaad et al. [22] for $O$. dillenii.

The saponification values of the studied samples ranging from 210.37 to 252.45 were higher than those of O. stricta and O. ficus-indica reported by Ennouri et al. [29], but they always stayed in the range of Codex Stan 210-1999 and COI standards. This parameter indicates the presence of saturated fatty acids in the studied oil, and a low value points to the predominance of long-chain fatty acids, as suggested by Akintayo and Bayer [34]. The refractive index is another parameter that can be used to measure the quality of the oil. Low indices characterize oils of high quality. The refractive indices of our oils were found to be higher compared to the results of El Mannoubi [33] and Ennouri [29], but in accordance with the norm (Codex Stan 210-1999 and COI standards). The density of the $O$. dillenii seed oils at $20^{\circ} \mathrm{C}$ compared favorably with that of $O$. stricta seed oil but was higher than that of O. ficus-indica seed oil [18]. Essaouira oil was found to be less acidic with a $\mathrm{pH}$ value of 6.96, higher than that of Oujda $(\mathrm{pH}=6.54)$ and Nador $(\mathrm{pH}=6.50)$ samples.

The evaluation of the acid value is another possible way to determine the quality of fatty acids present in the oil, which could in turn explain the shelf life and stability of such oil. Its value gives an idea of the free acid level. A value below 2 presents proof of good conservation (low amount of free acids) [32]. In the present work, the acid values found in $O$. dillenii seed oils for the three samples were higher than 2 . These high acidity levels could be attributed to the advanced maturity of the fruit and/or the harvesting period, as previously reported by Terouzi et al. [35]. This high acidity value could indicate strong enzymatic hydrolysis of the seeds during oil harvesting, handling, or processing [36]. It could also be explained by bad preservation. According to [37], when oil was subjected to poor storage conditions, its quality could deteriorate in various ways, but most often by hydrolysis or oxidation. The ester index test is an indirect method for determining the ester content of the oil. In fact, oils of excellent qualities have a large quantity of esters.

Generally, the studied oils extracted from $O$. dillenii seeds have been found to meet all quality criteria that confirm an excellent application. However, the observed differences between the samples could be due to geographical location, soil, climatic conditions, and maturation [38].

3.3. Chemical Composition of Hexane and Ethyl Acetate O. dillenii Extracts. In this study, we compared the yields and the chemical composition of the hexane and ethyl acetate extracts of skin, juice, and seeds of $O$. dillenii fruits harvested from three geographical locations in Morocco (Tables 4-6).

In the case of Nador variety, the hexane seed extract showed the highest yield with a value of $13.12 \%$, followed by Oujda extract (9.25\%) and then Essaouira extract (5.85\%). However, in the literature, Liu et al. [39] obtained a lower yield of $6.65 \%$ by using a supercritical carbon dioxide extraction of seed oil. Recently, Alsaad et al. [22] reported relatively the same yield of $6.5 \%$ by hydrodistillation extraction. Labuschagne and Hugo [40] reported that the oil content in cactus Opuntia dillenii seeds from South Africa was $5.69 \%$, while Chang [21] reported that the oil content in cactus pear seeds from China was $6.01 \%$.

The preliminary report of gas-chromatography coupled with mass spectrometry analysis of the hexane extract revealed the presence of numerous fatty acids. Their amount varied according to the locality and the part of cactus fruit. 
TAble 3: Physicochemical properties of $O$. dillenii seed oils from the three localities.

\begin{tabular}{lcccc}
\hline Properties & \multicolumn{2}{c}{$\begin{array}{c}\text { Locality } \\
\text { Nador }\end{array}$} & Essaouira & Codex Stan 210-1999 and COI standards [26] \\
\hline Acid value $(\mathrm{mg} \mathrm{KOH} / \mathrm{g}$ of oil) & $5.61 \pm 0.04$ & $7.85 \pm 0.20$ & $8.97 \pm 0.41$ & - \\
Saponification value $(\mathrm{mg}$ of KOH/g of oil) & $238.42 \pm 1.73$ & $252.45 \pm 1.54$ & $210.37 \pm 0.98$ & $168-265$ \\
Ester value $(\mathrm{mg} \mathrm{KOH} / \mathrm{g}$ of oil) & $232.82 \pm 1.92$ & $244.60 \pm 1.34$ & $201.40 \pm 1.63$ & - \\
Peroxide value $\left(\mathrm{Meq} \mathrm{O}_{2} / \mathrm{kg}\right.$ of oil) & $5.85 \pm 0.46$ & $6.35 \pm 0.92$ & $5.60 \pm 0.91$ & Max $(10)$ \\
Density $\left(20^{\circ} \mathrm{C}\right)$ & $0.926 \pm 0.04$ & $0.925 \pm 0.18$ & $0.913 \pm 0.37$ & $0.881-0.927$ \\
Refractive index $\left(20^{\circ} \mathrm{C}\right)$ & $1.476 \pm 0.93$ & $1.478 \pm 0.82$ & $1.477 \pm 0.62$ & $1.447-1.477$ \\
Water content $(\%)$ & $0.148 \pm 0.03$ & $0.189 \pm 0.09$ & $0.154 \pm 0.10$ & Max $0.2 \%$ \\
pH & $6.54 \pm 0.4$ & $6.50 \pm 0.34$ & $6.96 \pm 0.94$ & - \\
\hline
\end{tabular}

TABle 4: Chemical composition of hexane and ethyl acetate extracts from $O$. dillenii seeds fruits.

\begin{tabular}{|c|c|c|c|c|c|c|c|}
\hline \multirow{3}{*}{ Compounds } & \multirow{3}{*}{ Retention time (min) } & \multicolumn{6}{|c|}{ Relative content (\%) in seeds } \\
\hline & & \multicolumn{2}{|c|}{ Oujda } & \multicolumn{2}{|c|}{ Nador } & \multicolumn{2}{|c|}{ Essaouira } \\
\hline & & H. E & EAc. E & H. E & EAc. E & H. E & EAc. E \\
\hline Butanoic acid (C4:0) & $12.53 \pm 0.04$ & nd & nd & nd & $21.68 \pm 0.02$ & nd & nd \\
\hline Acetic acid & $12.89 \pm 0.04$ & nd & nd & nd & $7.37 \pm 0.11$ & nd & nd \\
\hline Capric acid (C10:0) & $14.69 \pm 0.04$ & nd & nd & nd & $4.41 \pm 0.03$ & nd & nd \\
\hline 2,4-Decadienal & $15.08 \pm 0.03$ & $0.71 \pm 0.12$ & $0.95 \pm 0.05$ & nd & $10.26 \pm 0.05$ & nd & $1.28 \pm 0.07$ \\
\hline Myristic acid (C14:0) & $20.40 \pm 0.02$ & $2.91 \pm 0.13$ & $0.75 \pm 0.12$ & $2.41 \pm 0.07$ & $1.58 \pm 0.08$ & $0.97 \pm 0.02$ & $0.54 \pm 0.12$ \\
\hline Methoxyacetic acid & $22.58 \pm 0.01$ & nd & nd & nd & nd & nd & $1.14 \pm 0.45$ \\
\hline Palmitic acid (C16:0) & $22.62 \pm 0.06$ & $24.27 \pm 0.17$ & $9.40 \pm 0.08$ & $21.50 \pm 0.41$ & $12.98 \pm 0.07$ & $19.81 \pm 0.07$ & $20.17 \pm 0.18$ \\
\hline Margaric acid $(\mathrm{C} 17: 0)$ & $23.10 \pm 0.01$ & nd & $6.18 \pm 0.33$ & nd & nd & nd & nd \\
\hline Oleic acid $(\mathrm{C} 18: 1)$ & $24.41 \pm 0.02$ & nd & $5.04 \pm 0.04$ & nd & nd & nd & nd \\
\hline Linoleic acid (C18:2) & $24.45 \pm 0.08$ & $66.57 \pm 0.08$ & $58.50 \pm 0.21$ & $72.39 \pm 0.11$ & $36.21 \pm 0.33$ & $69.60 \pm 1.01$ & $58.32 \pm 2.04$ \\
\hline Stearic acid $(\mathrm{C} 18: 0)$ & $24.77 \pm 0.05$ & $5.49 \pm 0.17$ & $19.1 \pm 0.40$ & $2.37 \pm 0.14$ & $5.29 \pm 0.12$ & $9.61 \pm 0.81$ & $18.04 \pm 0.21$ \\
\hline $\mathrm{SFA}^{\mathrm{a}}$ & & 32.67 & 35.43 & 26.28 & 45.94 & 30.39 & 39.89 \\
\hline $\mathrm{UFA}^{\mathrm{b}}$ & & 66.57 & 63.54 & 72.39 & 36.21 & 60.69 & 58.32 \\
\hline $\mathrm{UFA}^{\mathrm{S}} \mathrm{SF}^{\mathrm{c}}$ & & 2.04 & 1.79 & 2.75 & 0.78 & 2.29 & 1.46 \\
\hline Yield of extracts \% & & $9.25 \pm 0.07$ & $0.92 \pm 0.04$ & $13.12 \pm 0.02$ & $0.96 \pm 0.20$ & $5.85 \pm 0.47$ & $1.34 \pm 0.20$ \\
\hline
\end{tabular}

nd: not detected; H. E: hexane extract; EAc. E: ethyl acetate extract; a: saturated fatty acids (SFA); b: unsaturated fatty acids (UFA); c: unsaturation ratio = UFA/SFA.

The major constituents of the seed oils were linoleic acid with amounts varying from 66.57 to $72.39 \%$, followed by palmitic acid (19.81 to $24.27 \%)$, stearic acid (2.37 to $9.61 \%)$, and myristic acid (0.97 to $2.91 \%)$. The Nador locality oil presented the highest percentage of linoleic acid $(72.39 \%)$ followed by Essaouira and Oujda localities with 69.60 and $66.57 \%$, respectively. A high unsaturated ratio of 2.75 was detected for the Nador locality oil, followed by Essaouira and Oujda localities, 2.29 and 2.04, respectively. These values showed that the seed oils were rich in unsaturated fatty acids and confirmed their importance as nutritional value for human consumption.

In contrast, a very low amount of oil was recovered in the case of ethyl acetate seed extracts. The yield was about $1.35 \%$ for Essaouira, followed by 0.96 and $0.92 \%$ for Nador and Oujda localities, respectively. This oil was also dominated by linoleic acid whose values ranged from 36.21 to $72.39 \%$, followed by butanoic acid, 0 to $21.68 \%$, stearic acid, 5.49 to $23.25 \%$, and palmitic acid, 9.40 to $20.17 \%$. Oujda and Essaouira extracts were richer in linoleic acid, with a value of 72.39\%, than Nador extract with $36.21 \%$. However, a moderate quantity of butanoic acid as saturated fatty acid was found only in Nador extract with approximately $21.68 \%$. The ethyl acetate seed extract showed the presence of less than $5.04 \%$ of oleic unsaturated fatty acid in only Oujda locality and a low amount of margaric acid (6.18\%) in the same locality. On the other hand, the extract from Nador showed a higher value of unsaturated ratio (2.75), followed by Essaouira extract (2.29) and Oujda extract (2.04). Our results are in concordance with those reported in the literature by Alsaad et al. [22]. The major constituents of the seed oil were linoleic acid (72.9\%), palmitic acid (15.12\%), and stearic acid (7.51\%). However, Liu et al. [39] reported a different profile of the Chinese seed oil. The main components obtained were linolenic acid $(66.56 \%)$, palmitic acid (19.78\%), stearic acid (9.01\%), and linoleic acid (2.65\%). This variation could be due to geographical location, soil, climatic conditions, and maturation.

The yields of hexane and ethyl acetate extracts of $O$. dillenii juice from the three localities presented low values less than $0.86 \%$. The chemical composition analysis of hexane extract (Table 5) showed that palmitic acid was the dominant fatty acid with a value of $55.89 \%$ for Oujda sample followed by linoleic (39.09\%), oleic (24.82\%), and stearic acid with less than $4.47 \%$. 
TABle 5: Chemical composition of hexane and ethyl acetate extracts from $O$. dillenii juice fruits.

\begin{tabular}{|c|c|c|c|c|c|c|c|}
\hline \multirow{3}{*}{ Compounds } & \multirow{3}{*}{ Retention time (min) } & \multicolumn{6}{|c|}{ Relative content (\%) in juice } \\
\hline & & \multicolumn{2}{|c|}{ Oujda } & \multicolumn{2}{|c|}{ Nador } & \multicolumn{2}{|c|}{ Essaouira } \\
\hline & & H. E & EAc. E & H. E & EAc. E & H. E & EAc. E \\
\hline 1,4-Butanedioic acid & $12.68 \pm 0.03$ & nd & nd & nd & $1.08 \pm 0.01$ & nd & nd \\
\hline Acetic acid & $12.89 \pm 0.03$ & nd & nd & nd & nd & nd & $2.01 \pm 0.10$ \\
\hline Capric acid $(\mathrm{C} 10: 0)$ & $14.95 \pm 0.03$ & nd & nd & $2.29 \pm 0.02$ & nd & nd & $0.85 \pm 0.09$ \\
\hline Citric acid & $17.23 \pm 0.04$ & nd & $10.18 \pm 0.01$ & $1.61 \pm 0.03$ & $2.55 \pm 0.03$ & $3.49 \pm 0.02$ & $17.01 \pm 0.21$ \\
\hline Lauric acid $(\mathrm{C} 12: 0)$ & $17.89 \pm 0.04$ & $3.96 \pm 0.41$ & $1.64 \pm 0.31$ & nd & $2.45 \pm 0.05$ & $1.62 \pm 0.04$ & $7.61 \pm 0.17$ \\
\hline 9-Eicosene & $18.74 \pm 0.01$ & $4.69 \pm 0.01$ & nd & $1.18 \pm 0.01$ & nd & nd & nd \\
\hline Lauryl ethoxylate & $20.32 \pm 0.01$ & $2.76 \pm 0.51$ & $2.08 \pm 0.17$ & $1.6 \pm 0.05$ & nd & nd & nd \\
\hline Myristic acid (C14:0) & $20.40 \pm 0.02$ & nd & nd & nd & nd & $4.04 \pm 0.08$ & $12.54 \pm 0.28$ \\
\hline 11-Hexadecenoic acid (C16:1) & $22.42 \pm 0.01$ & nd & nd & nd & nd & $4.73 \pm 0.07$ & nd \\
\hline Palmitic acid $(\mathrm{C} 16: 0)$ & $22.96 \pm 0.06$ & $55.89 \pm 0.31$ & $6.81 \pm 0.11$ & $31.46 \pm 0.11$ & $17.77 \pm 0.24$ & $23.56 \pm 0.41$ & $9.65 \pm 0.08$ \\
\hline Margaric acid (C17:0) & $23.00 \pm 0.01$ & $0.15 \pm 0.17$ & $37.41 \pm 0.08$ & nd & $13.18 \pm 0.02$ & nd & $34.15 \pm 0.09$ \\
\hline Oleic acid $(\mathrm{C} 18: 1)$ & $23.89 \pm 0.03$ & $1.01 \pm 0.11$ & nd & $22.57 \pm 0.17$ & nd & $24.82 \pm 0.32$ & $5.15 \pm 0.09$ \\
\hline Linoleic acid $(\mathrm{C} 18: 2)$ & $24.39 \pm 0.08$ & $31.31 \pm 0.21$ & $16.04 \pm 0.25$ & $39.09 \pm 0.22$ & $47.13 \pm 0.35$ & $32.84 \pm 1.01$ & $10.46 \pm 0.21$ \\
\hline Stearic acid $(\mathrm{C} 18: 0)$ & $24.75 \pm 0.02$ & nd & $23.25 \pm 0.08$ & nd & $13.11 \pm 0.06$ & $4.47 \pm 0.09$ & nd \\
\hline Campesterol & $25.44 \pm 0.01$ & nd & $2.48 \pm 0.01$ & nd & $2.15 \pm 0.02$ & nd & nd \\
\hline $\mathrm{SFA}^{\mathrm{a}}$ & & 59.85 & 69.11 & 33.75 & 46.51 & 33.69 & 55.15 \\
\hline $\mathrm{UFA}^{\mathrm{b}}$ & & 32.32 & 16.04 & 61.66 & 47.13 & 62.39 & 15.61 \\
\hline $\mathrm{UFA} \mathrm{SFA}^{\mathrm{c}}$ & & 0.53 & 0.23 & 1.96 & 1.01 & 1.85 & 0.27 \\
\hline Sterols & & 0 & 2.48 & 0 & 2.15 & 0 & 0 \\
\hline Yield of extracts (\%) & & $0.49 \pm 0.01$ & $0.27 \pm 0.08$ & $0.40 \pm 0.12$ & $0.46 \pm 0.07$ & $0.45 \pm 0.19$ & $0.86 \pm 0.08$ \\
\hline
\end{tabular}

nd: no detected; H. E: hexane extract; EAc. E: ethyl acetate extract; a: saturated fatty acids (SFA); b: unsaturated fatty acids (UFA); c: unsaturation ratio = UFA/ SFA.

TABLE 6: Chemical composition of hexane and ethyl acetate extracts from O. dillenii skin fruits.

\begin{tabular}{|c|c|c|c|c|c|c|c|}
\hline \multirow{3}{*}{ Compounds } & \multirow{3}{*}{ Retention time (min) } & \multicolumn{6}{|c|}{ Relative content (\%) in skin } \\
\hline & & \multicolumn{2}{|c|}{ Oujda } & \multicolumn{2}{|c|}{ Nador } & \multicolumn{2}{|c|}{ Essaouira } \\
\hline & & H. E & EAc. E & H. E & EAc. E & H. E & EAc. E \\
\hline Propanoic acid & $12.02 \pm 0.01$ & nd & nd & nd & $2.09 \pm 0.10$ & nd & nd \\
\hline Capric acid $(\mathrm{C} 10: 0)$ & $14.99 \pm 0.01$ & nd & nd & nd & nd & nd & $2.1 \pm 0.09$ \\
\hline Citric acid & $17.23 \pm 0.04$ & $0.89 \pm 0.10$ & nd & $0.64 \pm 0.02$ & nd & $2.18 \pm 0.09$ & nd \\
\hline Lauric acid (C12:0) & $17.89 \pm 0.01$ & $0.98 \pm 0.01$ & nd & $1.27 \pm 0.31$ & nd & $3.43 \pm 0.21$ & nd \\
\hline 4-Fluorobenzoic acid & $17.52 \pm 0.04$ & $2.14 \pm 0.2$ & $16.48 \pm .31$ & nd & $1.06 \pm 0.28$ & nd & $0.6 \pm 0.01$ \\
\hline 9-Eicosene (E) & $18.78 \pm 0.01$ & nd & nd & nd & nd & nd & $3.5 \pm 0.09$ \\
\hline Lauryl ethoxylate & $20.32 \pm 0.01$ & nd & $3.28 \pm 0.04$ & $1.33 \pm 0.09$ & nd & nd & nd \\
\hline Myristic acid (C14:0) & $20.38 \pm 0.02$ & $2.34 \pm 0.02$ & nd & nd & nd & $6.37 \pm 0.32$ & nd \\
\hline Pentadecanoic acid (C15:0) & $21.48 \pm 0.03$ & $5.08 \pm 0.09$ & nd & nd & nd & $1.88 \pm 0.51$ & nd \\
\hline 11-Hexadecenoic acid $(\mathrm{C} 16: 1)$ & $22.39 \pm$ & nd & nd & nd & nd & $15.39 \pm 1.26$ & nd \\
\hline Palmitic acid $(\mathrm{C} 16: 0)$ & $23.60 \pm 0.09$ & $28.15 \pm 0.31$ & $13.01 \pm 0.41$ & $45.28 \pm 0.28$ & $28.34 \pm 0.89$ & $17.49 \pm 0.75$ & $14.17 \pm 0.29$ \\
\hline Margaric acid (C17:0) & $23.77 \pm 0.07$ & $3.15 \pm 0.09$ & nd & nd & nd & nd & $10.7 \pm 0.35$ \\
\hline Oleic acid $(\mathrm{C} 18: 1)$ & $24.13 \pm 0.05$ & $16.67 \pm 0.23$ & nd & nd & nd & nd & nd \\
\hline Linoleic & $24.40 \pm 0.09$ & $40.55 \pm 1.56$ & $67.07 \pm 2.01$ & $51.39 \pm 0.91$ & $68.09 \pm 0.78$ & $52.98 \pm 0.51$ & $38.27 \pm 0.86$ \\
\hline Stearic acid $(\mathrm{C} 18: 0)$ & $24.98 \pm 0.01$ & nd & nd & nd & nd & nd & $3.22 \pm 0.03$ \\
\hline $\mathrm{SFA}^{\mathrm{a}}$ & & 39.70 & 13.01 & 46.55 & 28.34 & 29.17 & 25.39 \\
\hline $\mathrm{UFA}^{\mathrm{b}}$ & & 57.22 & 67.07 & 51.39 & 68.09 & 68.37 & 58.27 \\
\hline $\mathrm{UFA} / \mathrm{SFA}^{\mathrm{c}}$ & & 1.44 & 5.15 & 1.09 & 2.43 & 2.34 & 2.29 \\
\hline Yield of extracts (\%) & & $1.77 \pm 0.10$ & $0.98 \pm 0.10$ & $1.16 \pm 0.37$ & $0.91 \pm 0.47$ & $1.06 \pm 0.20$ & $1.11 \pm 0.37$ \\
\hline
\end{tabular}

nd: no detected; H. E: hexane extract; EAc. E: ethyl acetate extract; a: saturated fatty acids (SFA); b: unsaturated fatty acids (UFA); c: unsaturation ratio = UFA/ SFA.

In the ethyl acetate extract, linoleic acid was found to be the most abundant fatty acid detected with $47.13 \%$ in Nador juice extract followed by stearic (23.25\%) and palmitic acid (17.77\%). However, citric acid was found especially in ethyl acetate extract with a moderate percentage $(17.01 \%)$ in Essaouira locality.
Interesting, the study showed the presence of small quantities of campesterol, which is a well-known beneficial agent with cholesterol-lowering and anticarcinogenic properties [41], concentrated especially in ethyl acetate extracts of Oujda and Nador varieties with contents of $2.48 \%$ and $2.15 \%$. It is also 
important to note the presence of margaric acid in ethyl acetate extract with a moderate amount of $37.41 \%$ and $34.15 \%$ in Oujda and Essaouira extracts, respectively. This compound was detected in a low value of $13.18 \%$ in Nador variety and was only present in trace amount, $0.15 \%$, in the hexane extract of Oujda locality.

Concerning the skin extract, the highest yield of 1.77\% was detected in the hexane extract of Oujda variety. However, the yield obtained in the ethyl acetate was about $1 \%$ in all the localities. Similar to previous results of seeds and juice extracts, the skin extracts were also found to be rich in linoleic and palmitic acids (Table 6).

The highest percentage of linoleic acid, $68.09 \%$, was found in Nador ethyl acetate extract. This compound was also abundant in the hexane extract with a quantity of $52.98 \%$ in the Essaouira locality. This study also showed that the Nador variety contained a higher amount of palmitic acid, $45.28 \%$ and $28.34 \%$ in hexane and ethyl acetate extracts, respectively. In contrast, oleic acid (16.67\%) and 11hexadecenoic acid (15.39\%) were present, as unsaturated fatty acids, only in the hexane extracts of both samples of Oujda and Essaouira.

In addition, the 4-fluorobenzoic acid was found in the ethyl acetate extract, with a percentage of $16.48 \%$, as well as in the hexane extract, with a low content of $2.14 \%$, of Oujda sample. This compound was also detected in ethyl acetate extract of Nador and Essaouira samples but only in trace amounts.

Furthermore, a low quantity of margaric acid was identified only in the ethyl acetate extract of Essaouira. Pentadecanoic acid and margaric acid have a positive effect on health in several disease etiologies [42]. Holman [43] showed that both pentadecanoic and margaric acids have a role in reducing the development of multiple sclerosis, suggesting that fatty acids could increase membrane fluidity [44] to a similar degree to polyunsaturated fatty acids. Besides and based on previous studies, some of the constituents identified in $O$. dillenii fruits were biologically active compounds. They were proven to possess pharmacologic activities which may contribute to the healing potential of the plant. Linoleic acid has beneficial properties for the skin, and, for this purpose, it is widely used in the cosmetics and pharmaceutical industry. Stearic acid has neutral effects on the concentration of LDL cholesterol in blood serum and no cholesterol-lowering impact on human health [45]. Based on UFA/SFA ratio, it is essential to mention that there is a higher amount of unsaturated fatty acids (UFA) in the skin and seed parts of the studied $O$. dillenii fruits than the juice part, which was found to be richer in saturated fatty acids (SFA). The ethyl acetate extract of Oujda skin extract presented the highest unsaturated ratio of 5.15. However, the hexane extract was less rich in unsaturated acids. Particularly, the study reported that the amount of linoleic acid is higher in comparison with other vegetable oils such as potato oil (52.69\%), pomegranate oil (3.84\%), and sesame oil (44.5\%). These findings were in accordance with recently published studies by Ghazi, where linoleic acid was the dominant fatty acid with an exceptional level up to $79.83 \%$ [46-49]. Finally, the evaluation of the chemical composition as well as the physicochemical properties of $O$. dillenii fruit extracts demonstrated that this plant could be an interesting natural source of edible oil containing high amounts of unsaturated fatty acids. In addition, it is interesting to note that the difference between the chemical compositions of the different extracts from the three varieties of $O$. dillenii fruits could be a consequence of many factors such as the degree of maturation, the growing conditions, and the nature of the solvent used during the extraction $[35,36]$. On the other hand, further detailed analyses and studies, along with isolation of active constituents, are needed to investigate this plant further.

3.4. Statistical Analyses. The statistical study was based on the hierarchical cluster analysis (HCA) and principal component analysis (PCA). The intermediate correlation matrices, correlation coefficients between the variables and the two axes $\mathrm{F} 1$ and $\mathrm{F} 2$, and the projection of the variables in the space of the axes F1 and F2 were obtained with XLSTAT software.

From the dendrogram produced by HCA (Figure 3), based on the Euclidean distances between collected samples, O. dillenii populations can be classified into five main clusters, which shows that there is a significant difference in the composition of $O$. dillenii extract harvested from three Moroccan regions (Oujda, Nador, and Essaouira). The samples linked by short distances are more similar than those connected by long ones. In the closer distances (18 units), the populations examined were divided into five groups (Gr 1 to 5). The first group (Cluster I), represented by five samples from the three regions of Morocco (Oujda, Nador, and Essaouira), SO1, SN1, SKN2, SKO2, and SE1, was rich in linoleic acid (32.27-72.39\%). These populations in the vicinity have a relatively different climate and soil, resulting in different oil contents. The second group (Cluster II), containing the following chemotypes: SN2, JN2, SKE1, SO2, and SE2, has been characterized by a significant percentage of linoleic acid (58.32-58.50\%). The ethyl acetate juice extracts from Oujda and Essaouira samples (JE2 and JO2) were found to construct the third group (Cluster III). This latter group was found to be rich in margaric acid (34.15-37.41\%), citric acid (10.18-17.01\%), and stearic acid (5.29-13.11\%). In the fourth group (Cluster IV), which was represented by SKE2, SKO1, JN1, and JE1 samples, palmitic acid (14.17-28.46\%), oleic acid (5.15-22.57\%), and linoleic acid (58.32-58.50\%) were found to be the most abundant components. The group also contains significant to moderate levels of oleic acid (5.04-16.67\%). Finally, the fifth group (Cluster V), represented by the two samples JO1 and SKN1, was found to be rich in linoleic acid (30.31-50.39\%) and palmitic acid (45.28-55.89\%).

The analysis of the results showed that most of the information is explained by the first two factorial axes. In the F1 $\times$ F2 factorial design, the eigenvalues of the two components F1 and F2 and their contribution to the total inertia are represented in Table 7. For the PCA, it was found that the first two main components accounted for $56.21 \%$ of the phytochemical variance. As shown in Figure 4, by considering the PCA1 axis, it is possible to note that the extracts of O. dillenii, JO2, JE2, and JN2, are distinguished by a greater quantity of margaric acid (0.854), citric acid (0.977), and stearic acid (0.147). On the other hand, the extracts SN1, SO1, SE1, SE2, SN2, SO2, SE2, SO2, SKN2, SKE1, and JN2 are characterized by a low content of these constituents. Meanwhile, SN1, SO1, SE1, SE2, SN2, SO2, SE2, 


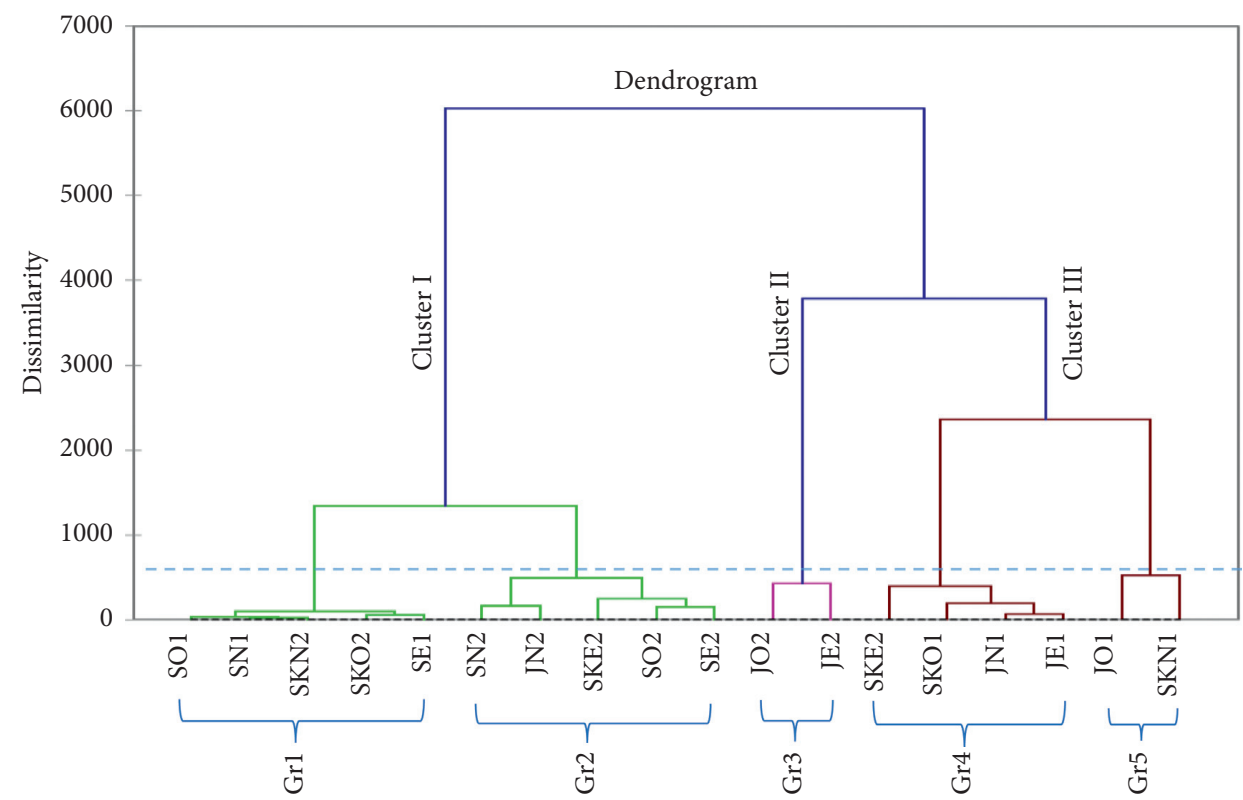

FIgURE 3: Dendrogram of $O$. dillenii extracts produced by the hierarchical cluster analysis.

TABLe 7: Distribution of inertia between the two axes $(\mathrm{F} 1 \times \mathrm{F} 2)$.

\begin{tabular}{lcc}
\hline & F1 & F2 \\
\hline Eigenvalue & 3.502 & 2.120 \\
Variability (\%) & 35.016 & 21.196 \\
Cumulative variance (\%) & 35.016 & 56.212 \\
\hline
\end{tabular}
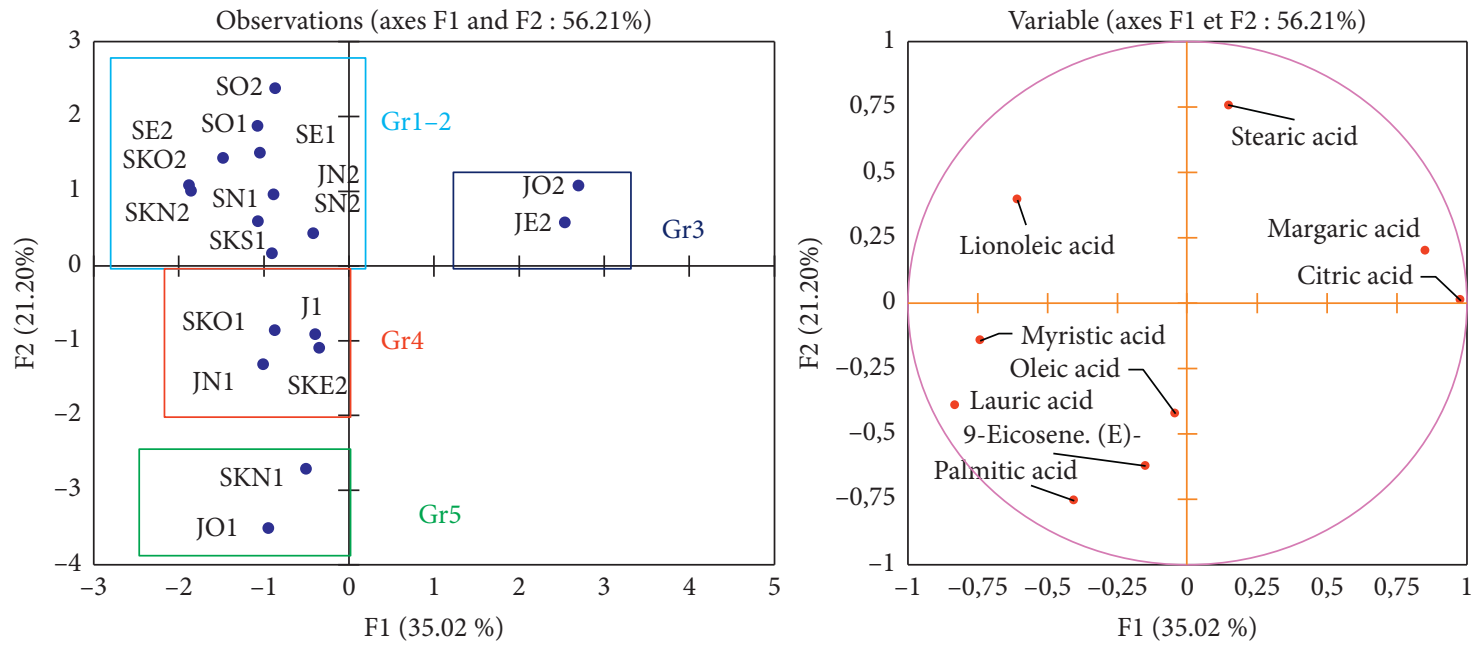

FIgURE 4: Principal component analysis of the composition variables of O. Dillenii extracts collected in the different regions of Morocco.

SO2, SKN2, SKE1, and JN2 are aligned on the PCA2 axis, where the most correlated variable is linoleic acid, which means that these extracts are rich in linoleic acid, and the reverse is true for JO2 and JE2. Table 8 shows that the higher the correlation is, the more the variable is related to the component. With the inertia of $21.02 \%$, the second component (F2) was negatively correlated with palmitic acid (0.754) which existed in moderate to large quantities in the different $O$. dillenii extracts collected from three different regions (SKN1 and JO1). These PCA data lead to the hierarchical upward classification of the AHA into four main groups that represent the following chemotypes: citric acid (0.977) (JO2 and JS2), myristic acid (0.738) (JN1, JS1, SKS2, and PO1), palmitic acid (0.754) (SKN1 and JO1), and linoleic acid (0.610) (SN1, SO1, SE1, SN2, SO2, SE2, SKO2, SKN2, SKS1, and JN2); their correlation matrices are represented in Table 9. This classification confirmed the results of HCA. 
TABLE 8: Correlation coefficients between variables and main axes.

\begin{tabular}{lcc}
\hline & F1 & F2 \\
\hline Citric acid & 0.977 & -0.008 \\
Lauric acid & -0.832 & -0.390 \\
9-Eicosene & -0.150 & -0.623 \\
Myristic acid & -0.738 & -0.141 \\
Palmitic acid & -0.405 & -0.754 \\
Margaric acid & 0.854 & 0.201 \\
Oleic acid & -0.040 & -0.420 \\
Linoleic acid & -0.610 & 0.397 \\
Stearic acid & 0.147 & 0.755 \\
\hline
\end{tabular}

TABLE 9: Correlation matrix between the variables.

\begin{tabular}{|c|c|c|c|c|c|c|c|c|c|}
\hline Variables & Citric acid & Lauric acid & $\begin{array}{c}9- \\
\text { Eicosene }\end{array}$ & Myristic acid & Palmitic acid & Margaric acid & Oleic acid & Linoleic acid & Stearic acid \\
\hline Citric acid & 1 & & & & & & & & \\
\hline Lauric acid & 0.794 & 1 & & & & & & & \\
\hline 9-Eicosene & -0.182 & 0.080 & 1 & & & & & & \\
\hline Myristic acid & 0.701 & 0.754 & -0.264 & 1 & & & & & \\
\hline Palmitic acid & -0.368 & 0.023 & 0.461 & -0.299 & 1 & & & & \\
\hline Margaric acid & 0.844 & 0.546 & 0.016 & 0.350 & -0.497 & 1 & & & \\
\hline Oleic acid & 0.033 & -0.068 & 0.328 & 0.039 & 0.046 & -0.002 & 1 & & \\
\hline Linoleic acid & -0.574 & -0.547 & -0.220 & -0.184 & -0.180 & -0.554 & -0.210 & 1 & \\
\hline Stearic acid & 0.131 & -0.187 & -0.240 & -0.263 & -0.458 & 0.416 & -0.271 & -0.022 & 1 \\
\hline
\end{tabular}

\section{Conclusion}

In this work, the GC-MS analysis showed that the studied extracts of $O$. dillenii fruit from the three localities contained a high percentage of unsaturated fatty acids and a low percentage of saturated fatty acids. Linoleic acid was the major fatty acid detected with a maximum content of $72.39 \%$, followed by palmitic acid (maximum value of $55.89 \%$ ). Otherwise, the juice extract of Oujda locality was richer in margaric acid (37.41\%), followed by the Essaouira skin extract (10.7\%) and Oujda seed extract (6.18\%). However, the campesterol was detected only in trace in the juice extract. The physicochemical properties of $O$. dillenii seed oils such as acid value, peroxide value, ester value, $\mathrm{pH}$ value, saponification value, density, and refractive index were all found to be in good accordance with the quality criteria for pure and fresh oils, which opens the way for their use in various cosmetic applications and as sources of dietary lipids. Moreover, the presence of campesterol in the juice extract demonstrated that the $O$. dillenii fruits contained nutrients that are nutritionally beneficial to human health. Besides, the study also indicated that the chemical variation between the different extracts of $O$. dillenii depended especially on the environmental factors, climatic and geographical conditions, and the domestication of the species. Furthermore, statistical methods such as principal component analysis (PCA) and hierarchical cluster analysis (HCA) were employed based on the obtained results to highlight the existing correlation between the different parameters and the distribution of the variables in groups.

\section{Data Availability}

All data generated or analyzed during this study are included within this article.

\section{Conflicts of Interest}

The authors declare that they have no conflicts of interest.

\section{Acknowledgments}

The authors gratefully acknowledge the financial support for this study by the National Center for Scientific and Technical Research (CNRST) of Morocco (PPR2).

\section{References}

[1] J. Katanic, F. Yousfi, M. C. Caruso et al., "Characterization of bioactivity and phytochemical composition with toxicity studies of different Opuntia dillenii extracts from Morocco," Food Bioscience, vol. 30, Article ID 100410, 2019.

[2] M. S. Ahmed, N. D. E. Tanbouly, W. T. Islam, A. A. Sleem, and A. S. E. Senousy, "Antiinflammatory flavonoids fromOpuntia dillenii (Ker-Gawl) Haw. flowers growing in Egypt," Phytotherapy Research, vol. 19, no. 9, pp. 807-809, 2005.

[3] I. Z. A. Abdallah, "Evaluation of hypoglycemic activity of Opuntia dillenii haw fruit juice in streptozotocin-induced diabetic rats," The Egyptian Journal of Hospital Medicine, vol. 33, no. 1, pp. 544-558, 2008.

[4] Z. Bouzoubaa, Y. Essoukrati, S. Tahrouch, A. Hatimi, S. Gharby, and H. Harhar, "Phytochemical study of prickly 
pear from southern Morocco," Journal of the Saudi Society of Agricultural Sciences, vol. 15, no. 2, pp. 155-161, 2016.

[5] B. Hartmut, "Opuntia dillenii-an interesting and promising Cactaceae taxon," Journal of the Professional Association for Cactus Development, vol. 10, pp. 148-170, 2008.

[6] R. Ciriminna, R. Delisi, L. Albanese, F. Meneguzzo, and M. Pagliaro, "Opuntia ficus-indica seed oil: biorefinery and bioeconomy aspects," European Journal of Lipid Science and Technology, vol. 119, no. 8, Article ID 1700013, 2017.

[7] B. Amna, U. Saleem, U. H. Hasan, F. Abid, and A. M. Uttra, "Hyperlipidemia and hypertension; cardiovascular risk factors, various induction methods and their management by ethnomedicines," International Research Journal of Pharmacy, vol. 7, no. 11, pp. 1-9, 2016.

[8] R. Saleem, M. Ahmad, A. Azmat et al., "Hypotensive activity, toxicology and histopathology of opuntioside-I and methanolic extract of Opuntia dillenii," Biological and Pharmaceutical Bulletin, vol. 28, no. 10, pp. 1844-1851, 2005.

[9] M. J. Cejudo-Bastante, M. Chaalal, H. Louaileche, J. Parrado, and F. J. Heredia, "Betalain profile, phenolic content, and color characterization of different parts and varieties of Opuntia ficus-indica," Journal of Agricultural and Food Chemistry, vol. 62, no. 33, pp. 8491-8499, 2014.

[10] G. Y. Attia, M. E. M. Moussa, and E. R. Sheashea, "Characterization of red pigments extracted from red beet (Beta vulgaris L.) and its potential uses as antioxidant and natural food colorants," Egyptian Journal of Agricultural Research, vol. 91, no. 3, pp. 1095-1110, 2013.

[11] C. Saenz, "Cactus pear fruits and cladodes: a source of functional components for foods," Acta Horticulturae, vol. 581, no. 581, pp. 253-263, 2002.

[12] M. I. Khan and P. Giridhar, "Plant betalains: chemistry and biochemistry," Phytochemistry, vol. 117, pp. 267-295, 2015.

[13] D. Butera, L. Tesoriere, F. Di Gaudio et al., "Antioxidant activities of Sicilian prickly pear (Opuntia ficus indica) fruit extracts and reducing properties of its betalains: betanin and indicaxanthin," Journal of Agricultural and Food Chemistry, vol. 50, no. 23, pp. 6895-6901, 2002.

[14] S. Cody and L. M. Jeanne, "Improved efficiency of betaninbased dye-sensitized solar cells," Journal of Photochemistry and Photobiology A: Chemistry, vol. 221, no. 1, pp. 90-97, 2011.

[15] M. Perfumi and R. Tacconi, "Antihyperglycemic effect of fresh Opuntia dillenii fruit from tenerife (canary islands)," International Journal of Pharmacognosy, vol. 34, no. 1, pp. 41-47, 1996.

[16] E.-H. Park, J.-H. Kahng, S. H. Lee, and K.-H. Shin, “An antiinflammatory principle from cactus," Fitoterapia, vol. 72, no. 3, pp. 288-290, 2001.

[17] A. P. Sharavana Kumaar, "Antibacterial and antifungal activity of Opuntia dillenii (Cactaceae) fruit extract," Journal of Envirenement Nanotechnol, vol. 2, no. 1, pp. 16-19, 2013.

[18] J. M. Feugang, "Nutritional and medicinal use of Cactus pear (Opuntia spp.) cladodes and fruits," Frontiers in Bioscience, vol. 11, no. 1, pp. 2574-2589, 2006.

[19] E. M. Galati, M. R. Mondello, E. R. Lauriano, M. F. Taviano, M. Galluzzo, and N. Miceli, "Opuntia ficus indica (L.) Mill. fruit juice protects liver from carbon tetrachloride-induced injury," Phytotherapy Research, vol. 19, no. 9, pp. 796-800, 2005.

[20] M. Bouhrim, N. E. Daoudi, H. Ouassou et al., "Phenolic content and antioxidant, antihyperlipidemic, and antidiabetogenic effects of Opuntia dillenii seed oil," The Scientific World Journal, vol. 2020, Article ID 5717052, 8 pages, 2020.
[21] S.-F. Chang, C.-L. Hsieh, and G.-C. Yen, "The protective effect of Opuntia dillenii Haw fruit against low-density lipoprotein peroxidation and its active compounds," Food Chemistry, vol. 106, no. 2, pp. 569-575, 2008.

[22] A. J. A. Alsaad, A. B. Altemimi, S. N. Aziz, and N. Lakhssassi, "Extraction and identification of cactus Opuntia dillenii seed oil and its added value for human health benefits," Pharmacognosy Journal, vol. 11, no. 3, pp. 579-587, 2019.

[23] M. Bouhrim, "Diabetic rats," Asian Pacific Journal of Tropical Biomedicine, vol. 9, no. 9, p. 381, 2019.

[24] M. Bouhrim, "Hepatoprotective effect of Opuntia dillenii seed oil on $\mathrm{CCl} 4$ induced acute liver damage in rat," Asian Pacific Journal of Tropical Biomedicine, vol. 8, no. 5, p. 254, 2018.

[25] C. A. Commission, Codex Standard for Infant Formula Codex Stan 72-1981 (Amended 1983, 1985, 1987, 1997)Food and Agriculture Organization of the United Nations and World Health Organization, Rome, Italy, 1981.

[26] C. Alimentarius, Codex Standard for Named Vegetable Oils, Codex Stan 210-1999, pp. 11-25, Codex Alimentarius, Rome, Italy, 2001.

[27] C. Alimentarius, Recomended International Standard for Concetrated Orange Juice Preserved Exclusively by Physical Process, Cac/acceptances/Part Irev. 2, 1 Feb 1983 Appendx II, 1983.

[28] D. V. Wotto, "Physico-chemical characterization of oil and defatted meal from anacardium occidentale acclimated to teval in northen Benin," World Journal of Pharmacy and Pharmaceutical Sciences, vol. 4, no. 11, pp. 1912-1920, 2015.

[29] M. Ennouri, B. Evelyne, M. Laurence, and A. Hamadi, "Fatty acid composition and rheological behaviour of prickly pear seed oils," Food Chemistry, vol. 93, no. 3, pp. 431-437, 2005.

[30] J. Arrizon, C. Calderon, and G. Sandoval, "Effect of different fermentation conditions on the kinetic parameters and production of volatile Compounds during the elaboration of a prickly pear distilled beverage," Journal of Industrial Microbiology and Biotechnology, vol. 33, no. 11, pp. 921-928, 2006.

[31] M. O. Aremu, "Effect of processing on nutritional composition of African locust bean (Parkia biglobosa) and mesquite bean (Prosopis africana) seeds," Communications in Applied Sciences, vol. 3, no. 1, pp. 22-41, 2015.

[32] N. U. Chinedu, A. Benjamin, and A. Peter, "Chemical composition and physicochemical analysis of matured stems of Opuntia dillenii grown in Nigeria," Food Science and Technology, vol. 5, no. 5, pp. 106-112, 2017.

[33] I. El Mannoubi, S. Barrek, T. Skanji, H. Casabianca, and H. Zarrouk, "Characterization of Opuntia ficus indica seed oil from Tunisia," Chemistry of Natural Compounds, vol. 45, no. 5, pp. 616-620, 2009.

[34] E. T. Akintayo and E. Bayer, "Characterisation and some possible uses of Plukenetia conophora and Adenopus breviflorus seeds and seed oils," Bioresource Technology, vol. 85, no. 1, pp. 95-97, 2002.

[35] W. Terouzi, "Comparative study of physical and chemical propriety of the oil of some varieties of olive trees," International Journal of Innovation and Applied Studies, vol. 6, no. 4, pp. 1096-1103, 2014.

[36] F. Brahmi, S. Haddad, K. Bouamara et al., "Comparison of chemical composition and biological activities of Algerian seed oils of Pistacia lentiscus L., Opuntia ficus indica (L.) mill. and Argania spinosa L. Skeels," Industrial Crops and Products, vol. 151, Article ID 112456, 2020. 
[37] N. A. Kandji, Etude de la composition chimique et de la qualité d'huiles végétales artisanales consommées au Sénégal, Thèse de pharmacie, Tours, France, 2001.

[38] C. Pereira, M. J. Serradilla, A. Martín, M. d. C. Villalobos, F. Pérez-Gragera, and M. López-Corrales, "Agronomic behaviour and quality of six fig cultivars for fresh consumption," Scientia Horticulturae, vol. 185, pp. 121-128, 2015.

[39] W. Liu, Y.-J. Fu, Y.-G. Zu et al., "Supercritical carbon dioxide extraction of seed oil from Opuntia dillenii Haw. and its antioxidant activity," Food Chemistry, vol. 114, no. 1, pp. 334-339, 2009.

[40] M. T. Labuschagne and A. Hugo, "Oil content and fatty acid composition of cactus pear seed compared with cotton and grape seed," Journal of Food Biochemistry, vol. 34, no. 1, pp. 93-100, 2010.

[41] Y. K. Tosun, "Relationship between plant sterols (b-sitositerol, campesterol, stigmasterol) and nutrients of bread wheat cultivars," Fresenius Environmental Bulletin, vol. 28, pp. 1707-1714, 2019.

[42] P. J. Meikle, "Plasma lipid profiling shows similar associations with prediabetes and type 2 diabetes," PLoS One, vol. 8, no. 9, Article ID e74341, 2013.

[43] R. T. Holman, S. B. Johnson, and E. Kokmen, "Deficiencies of polyunsaturated fatty acids and replacement by nonessential fatty acids in plasma lipids in multiple sclerosis," Proceedings of the National Academy of Sciences, vol. 86, no. 12, pp. 4720-4724, 1989.

[44] R. T. Holman, C. E Adams, R. A Nelson et al., "Patients with anorexia nervosa demonstrate deficiencies of selected essential fatty acids, compensatory changes in nonessential fatty acids and decreased fluidity of plasma lipids," The Journal of Nutrition, vol. 125, no. 4, pp. 901-907, 1995.

[45] M. Jalali, M. Karamizadeh, G. A. Ferns, M. Zare, S. P. Moosavian, and M. Akbarzadeh, "The effects of cashew nut intake on lipid profile and blood pressure: a systematic review and meta-analysis of randomized controlled trials," Complementary Therapies in Medicine, vol. 50, p. 102387, 2020.

[46] Z. Ghazi, "Fatty acids sterols and vitamin E composition of seed oil of Opuntia ficus indica and Opuntia dillenii from Morocco," Journal of Materials and Environmental Science, vol. 5, no. 6, pp. 967-972, 2013.

[47] M. S. Alkaltham, M. M. Özcan, N. Uslu, A. M. Salamatullah, and K. Hayat, "Characterization of oil uptake and fatty acid composition of pre-treated Potato slices fried in sunflower and olive oils," Journal of Oleo Science, vol. 69, no. 3, pp. 185-190, 2020.

[48] M. Parsaeian, M. Shahabi, and H. Hassanpour, "The integration of image processing and artificial neural network to estimate four fatty acid contents of sesame oil," $L W T$, vol. 129, p. 109476, 2020.

[49] L. D. O. Silva et al., "Pomegranate (Punica granatum L.) seed oil enriched with conjugated linolenic acid (cLnA), phenolic compounds and tocopherols: improved extraction of a specialty oil by supercritical CO2," The Journal of Supercritical Fluids, vol. 147, pp. 126-137, 2019. 